\title{
A Case of Ischemic Colitis Complicated by Clostridioides Difficile Infection Treated with Fecal Microbiota Transplantation
}

\author{
Seok Hyung Kang', Tae-Geun Gweon ${ }^{2}$, Hyunjung Hwang ${ }^{1}$ and Myong Ki Baeg ${ }^{1}$ \\ ${ }^{1}$ Division of Gastroenterology, Department of Internal Medicine, International St. Mary's Hospital, Catholic Kwandong University, College of \\ Medicine, Incheon, ${ }^{2}$ Division of Gastroenterology, Department of Internal Medicine, The Catholic University of Korea, College of Medicine, \\ Bucheon, Korea
}

Ischemic colitis is an inflammatory condition of the colon that results from insufficient blood supply commonly caused by enterocolitis, vessel occlusion, or shock. In contrast, pseudomembranous colitis is a clinical manifestation of Clostridioides difficile infection (CDI). Ischemic colitis caused by CDI has rarely been reported. Fecal microbiota transplantation (FMT) is an efficient treatment for refractory or fulminant CDI, and the indications for its use have recently expanded. However, performing FMT in patients with ischemic colitis is challenging because of the risk of perforation. Here, we have presented a case of ischemic colitis caused by CDI that was successfully treated with FMT via sigmoidoscopy. Clin Endosc 2022 Jan 20. [Epub ahead of print]

Key Words: Clostridioides difficile infection; Fecal microbiota transplantation; Ischemic colitis; Pseudomembranous colitis

\section{INTRODUCTION}

Clostridioides difficile infection (CDI) is a common diarrheal disease that can be induced by prolonged antibiotic use. Clinical manifestations of CDI vary according to comorbidities and region of infection. ${ }^{1}$ Ischemic colitis is an inflammatory condition of the large bowel that commonly results from insufficient blood supply due to enterocolitis, vessel occlusion, or shock. It has a different diagnosis from that of pseudomembranous colitis (PMC), which is a clinical manifestation of CDI. ${ }^{2}$ Ischemic colitis caused by CDI has been rarely reported. ${ }^{3,4}$ However, $C$. difficile was not the causative organism in these cases. Fecal microbiota transplantation (FMT) is highly efficacious for recurrent or refractory CDI. ${ }^{5-7}$ However, performing FMT

Received: July 5, 2021 Revised: August 24, 2021

Accepted: August 28, 2021

Correspondence: Tae-Geun Gweon

Division of Gastroenterology, Department of Internal Medicine, Bucheon St. Mary's Hospital, The Catholic University of Korea, College of Medicine, 327, Sosa-ro, Wonmi-gu, Bucheon 14647, Korea

Tel: +82-32-340-2258, Fax: +82-32-340-2255, E-mail: gweontae@naver.com ORCID: https://orcid.org/0000-0002-0884-7228

(c) This is an Open Access article distributed under the terms of the Creative Commons Attribution Non-Commercial License (http://creativecommons.org/ licenses/by-nc/3.0) which permits unrestricted non-commercial use, distribution, and reproduction in any medium, provided the original work is properly cited. in patients with ischemic colitis is challenging because of the risk of perforation. Here, we have presented a case of ischemic colitis caused by CDI that was successfully treated with FMT.

\section{CASE REPORT}

A 53-year-old woman without comorbidities presented to the emergency room of our hospital complaining of a headache. She was diagnosed with subarachnoid hemorrhage, for which she underwent coil embolization. During hospitalization, however, she developed pneumonia and was treated with mechanical ventilation. Methicillin-resistant Staphylococcus aureus was isolated from her blood culture, and third-generation cephalosporins and intravenous vancomycin were administered to treat the pneumonia. Diarrhea (5 times/day) and abdominal distension developed 2 weeks after the initiation of antibiotic treatment.

Sigmoidoscopy revealed yellowish plaques covering the sigmoid colon, suggesting the possibility of PMC (Fig. 1). Additionally, $C$. difficile toxins were detected and isolated from her stool samples. Therefore, oral metronidazole $(500 \mathrm{mg} \times$ 3 times/day) was administered for 14 days. After resolution of CDI, septic arthritis developed as a complication, thus, continuous administration of broad-spectrum antibiotics was 
needed. The diarrhea reoccurred and $C$. difficile toxins were detected in the stool. Oral vancomycin (125 mg) was administered four times daily to treat the recurrent CDI.

Seven days after vancomycin treatment, the patient developed hematochezia, prompting the increase in vancomycin dosage to $500 \mathrm{mg} \times 4$ times/day, supplemented with intravenous metronidazole (500 $\mathrm{mg} \times 3$ times/day). Subsequently, the patient developed abdominal distension and ileus, prompting insertion of a nasogastric tube for decompression. Four

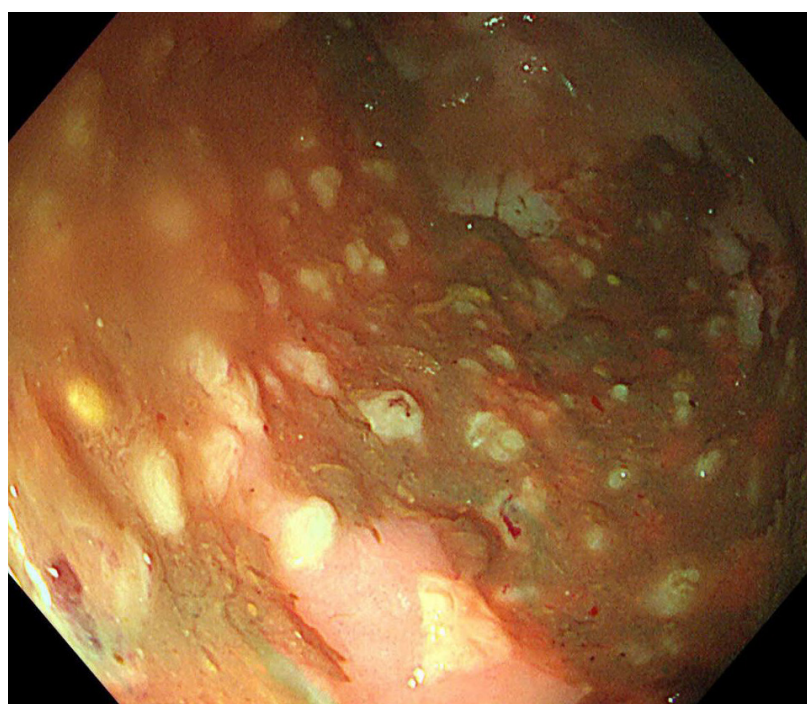

Fig. 1. Sigmoidoscopic finding. Sigmoidoscopy showing multiple yellowish plaques in the sigmoid colon. days after the change in medication, the volume and frequency of bloody diarrhea increased to 8 times/day. Sigmoidoscopy revealed multiple various-sized bullae and hemorrhages from the rectum to the sigmoid colon. Yellowish pseudomembranes were also observed in the bullae (Fig. 2).

Owing to the complications that arose despite the interventions, we established that the ongoing medical treatment for the patient's CDI was ineffective, leading us to perform FMT as an alternative treatment. After obtaining informed consent, FMT was performed on hospitalization day 70. Fecal matter for the FMT was obtained from the patient's 28-year-old son. Hepatitis B surface antigen, hepatitis C antibody, syphilis reagin test, $C$. difficile toxins, and human immunodeficiency virus were not detected in the stool sample. The donor's stool was collected 1 day before the FMT procedure and stored in a commercial freezer at $-20{ }^{\circ} \mathrm{C}$. A mixture containing 80 $\mathrm{g}$ of stool and $240 \mathrm{~mL}$ of normal saline (1:3) was placed in a commercial blender and ground for 4 minutes until no visible particles remained.

The patient's vital signs on the day of the FMT were as follows: blood pressure, 116/70 $\mathrm{mmHg}$; pulse rate, 90/min; and body temperature, $37.2^{\circ} \mathrm{C}$. The laboratory test results on the day of the FMT were as follows: hemoglobin, $10.9 \mathrm{~g} / \mathrm{dL}$; platelet count, $126 \times 10^{9} / \mathrm{L}$; white blood cell count, $13,200 / \mathrm{mm}^{3}$; C-reactive protein, $45.8 \mathrm{mg} / \mathrm{dL}$; albumin, $2.2 \mathrm{~g} / \mathrm{dL}$; and creatinine, $1.37 \mathrm{mg} / \mathrm{dL}$.

FMT was performed using sigmoidoscopy with $\mathrm{CO}_{2}$ gas to minimize inflation. Multiple various-sized bullae with hemorrhage were observed covering the rectum, sigmoid colon, and
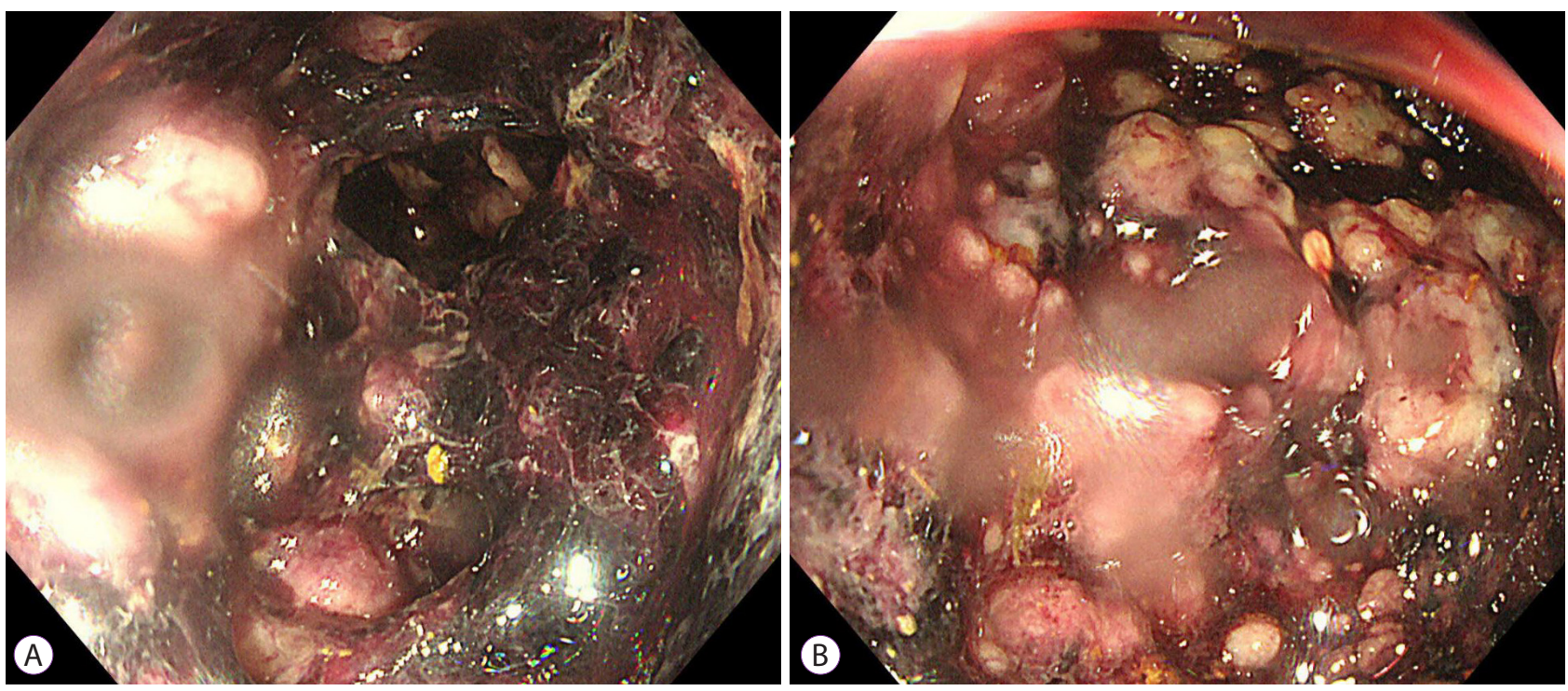

Fig. 2. Endoscopic image showing ischemic colitis. (A) Sigmoidoscopy showing multiple various-sized bullae with hemorrhage from the rectum to the distal transverse colon. (B) Focal yellowish pseudomembranes can been seen among the bullae. 


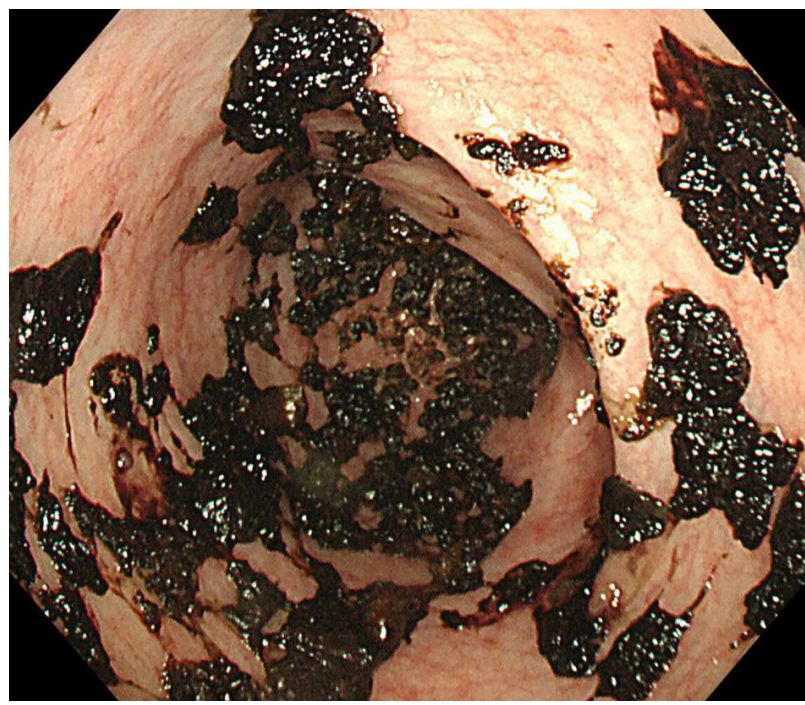

Fig. 3. Resolution of ischemic colitis. Sigmoidoscopy showing regression of hemorrhagic bullae to crusted blood. Majority of the colon mucosa has recovered.

descending colon. The colonoscope was inserted cautiously until the normal colonic mucosa was observable at a position that was thought to be the transverse colon. The fecal mixture was transferred to the recipient's intestines through the working channel of the endoscope. The procedure was performed with the patient in the right lateral position to prevent rapid evacuation of the fecal suspension. The total operation time was approximately 15 minutes; however, the patient was kept in the right lateral position for an additional hour. Oral vancomycin and intravenous metronidazole were continuously administered after FMT.

One day after the procedure, the patient's abdominal distension improved slightly, and the number of diarrhea episodes decreased to 3 times/day. Four days after FMT, the bloody diarrhea stopped, therefore, antibiotic therapy for CDI was discontinued. Sigmoidoscopy was performed without enema 5 days after the procedure and showed that all bullae had regressed to crusted blood (Fig. 3). The patient was discharged on hospitalization day 105, which was 35 days after FMT. This case is summarized in Table 1.

\section{DISCUSSION}

In this case report, we have described the use of FMT for the treatment of ischemic colitis caused by CDI. Despite the risk of perforation, FMT was performed through sigmoidoscopy because the patient developed abdominal distension and ileus. The patient was cured after only one session of FMT. To the best of our knowledge, successfully using FMT to treat ischemic colitis caused by CDI is a novel achievement in this field.

The presence of multiple, yellowish plaques in the colon is a typical endoscopic finding of PMC. ${ }^{1}$ Additionally, multiple bullae with hemorrhage were also observed in the patient's colon, suggesting that the PMC associated with CDI had progressed to ischemic colitis. However, it was difficult to determine whether the ischemic colitis was complicated by PMC because the yellowish plaques were found in small numbers. Most of the colon showed ischemic changes up to the descending colon. We suspected a relationship between CDI and ischemic colitis because C. difficile toxins were detected in the patient's stool.

Although there is no grading system for PMC, we believe that ischemic colitis represents its most severe form. Manifestations of severe, complicated CDI include shock and ileus. ${ }^{1}$ This patient developed ileus and had a risk of perforation because the colon mucosa was friable, a condition consistent

Table 1. Case Summary

\begin{tabular}{lcr}
\hline Hospital course & Event & Treatment \\
\hline Hospital day\#14 & CDI, first episode & Oral metronidazole \\
Hospital day\#34 & Septic arthritis & Broad spectrum antibiotics \\
Hospital day\#53 & CDI recurrence & Oral vancomycin $(125 \mathrm{mg} \times 4)$ \\
Hospital day\#60 & Symptom aggravation & Oral vancomycin $(500 \mathrm{mg} \times 4)$ and intravenous metronidazole \\
Hospital day\#64 & Sigmoidoscopy revealed ischemic colitis & Fecal microbiota transplantation \\
Hospital day\#70 & & \\
Hospital day\#74 & Diarrhea stop & \\
Hospital day\#74 & Improvement of ischemic colitis & \\
Hospital day\#105 & Discharge & \\
\hline
\end{tabular}

CDI, Clostridioides difficile infection. 
with severe CDI, which has a mortality rate as high as $50 \%{ }^{8}$ Recently, the indications for FMT have been expanded to include severe, complicated CDI that is refractory to medical treatment within 48 hours. ${ }^{5}$ FMT has favorable efficacy in the treatment of fulminant CDI.

Colonoscopic infusion is the preferred route for FMT, but alternative routes include the upper gastrointestinal (GI) tract or enema. ${ }^{10}$ The upper GI tract route can be chosen for unconscious patients or those with poor medical conditions. ${ }^{11}$ However, this route is contraindicated in patients with ileus drainage because of the risk of serious adverse events such as vomiting or aspiration. FMT can also be performed using enema, which is less invasive than colonoscopic or sigmoidoscopic infusion. Nonetheless, we did not consider enema in this case because the patient was critically ill and confused. Fecal suspension should be retained for at least 30 minutes after infusion, but the patient did not seem to be able to retain it because of her condition. Therefore, in this case report, we successfully treated fulminant CDI using sigmoidoscopic FMT. ${ }^{12}$

CDI is the most common etiology of PMC, and its incidence is positively correlated with the increase in population age and comorbidities. ${ }^{13}$ Manifestations of CDI have worsened and the recurrence rate has also escalated. ${ }^{1}$ The incidence of ischemic colitis caused by CDI is anticipated to increase in the future. Multiple FMTs can be considered in cases of early failure or ineffectiveness of the first FMT. ${ }^{14}$ We considered that the patient would possibly need multiple applications of FMT, but fortunately, she recovered after only a single session of FMT. Our report suggests that FMT can be considered an effective treatment option for patients with ischemic colitis complicated by CDI.

Conflict of Interest

The authors have no potential conflicts of interest.

Funding

This work was supported by the National Research Foundation of Korea(NRF) grant funded by the Korea government(MSIT) (grant number: NRF-2021R1G1A1094049).

\section{Author Contributions}

Conceptualization: Tae-Geun Gweon

Data curation: Hyunjung Hwang

Supervision: TGG, Myong Ki Baeg

Writing-original draft: Seok Hyung Kang
ORCID
Seok Hyung Kang

Tae-Geun Gweon

Hyunjung Hwang

Myong Ki Baeg https://orcid.org/0000-0003-3367-3941

https://orcid.org/0000-0002-0884-7228

https://orcid.org/0000-0003-3688-0962

https://orcid.org/0000-0002-4807-2447

\section{REFERENCES}

1. Cohen SH, Gerding DN, Johnson S, et al. Clinical practice guidelines for Clostridium difficile infection in adults: 2010 update by the society for healthcare epidemiology of America (SHEA) and the infectious diseases society of America (IDSA). Infect Control Hosp Epidemiol 2010;31:431455.

2. Trotter JM, Hunt L, Peter MB. Ischaemic colitis. BMJ 2016;355:i6600.

3. Moulis H, Vender RJ. Antibiotic-associated hemorrhagic colitis. J Clin Gastroenterol 1994;18:227-231.

4. Kendrick JB, Risbano M, Groshong SD, Frankel SK. A rare presentation of ischemic pseudomembranous colitis due to Escherichia coli O157:H7. Clin Infect Dis 2007;45:217-219.

5. Moore T, Rodriguez A, Bakken JS. Fecal microbiota transplantation: a practical update for the infectious disease specialist. Clin Infect Dis 2014;58:541-545.

6. Kelly CR, Ihunnah C, Fischer M, et al. Fecal microbiota transplant for treatment of Clostridium difficile infection in immunocompromised patients. Am J Gastroenterol 2014;109:1065-1071.

7. Gweon TG, Na SY. Next generation fecal microbiota transplantation. Clin Endosc 2021;54:152-156.

8. Jaber MR, Olafsson S, Fung WL, Reeves ME. Clinical review of the management of fulminant clostridium difficile infection. Am J Gastroenterol 2008;103:3195-3203; quiz 3204.

9. Fischer M, Sipe BW, Rogers NA, et al. Faecal microbiota transplantation plus selected use of vancomycin for severe-complicated Clostridium difficile infection: description of a protocol with high success rate. Aliment Pharmacol Ther 2015;42:470-476.

10. Gough E, Shaikh H, Manges AR. Systematic review of intestinal microbiota transplantation (fecal bacteriotherapy) for recurrent Clostridium difficile infection. Clin Infect Dis 2011;53:994-1002.

11. Gweon TG, Kim J, Lim CH, et al. Fecal microbiota transplantation using upper gastrointestinal tract for the treatment of refractory or severe complicated Clostridium difficile Infection in elderly patients in poor medical condition: the first study in an Asian country. Gastroenterol Res Pract 2016;2016:2687605.

12. Gweon TG, Lee KJ, Kang DH, et al. A case of toxic megacolon caused by clostridium difficile infection and treated with fecal microbiota transplantation. Gut Liver 2015;9:247-250.

13. Martin JSH, Monaghan TM, Wilcox MH. Clostridium difficile infection: epidemiology, diagnosis and understanding transmission. Nat Rev Gastroenterol Hepatol 2016;13:206-216.

14. Fischer M, Kao D, Mehta SR, et al. Predictors of early failure after fecal microbiota transplantation for the therapy of Clostridium difficile infection: a multicenter study. Am J Gastroenterol 2016;111:1024-1031. 